Article

\title{
On a One-Dimensional Hydrodynamic Model for Semiconductors with Field-Dependent Mobility
}

\author{
Giuseppe Alì $^{1,2, *(\mathbb{D})}$, Francesco Lamonaca ${ }^{3} \mathbb{D}$, Carmelo Scuro $^{1}$ id and Isabella Torcicollo $^{4}(\mathbb{D}$ \\ 1 Department of Physics, University of Calabria, 87036 Arcavacata, Italy; carmelo.scuro@unical.it \\ 2 National Institute for Nuclear Physics (INFN), Associated Group of Cosenza, Arcavacata di Rende, \\ 87036 Cosenza, Italy \\ 3 Department of Computer Science, Modelling, Electronic and Systems (DIMES), University of Calabria, \\ 87036 Arcavacata, Italy; f.lamonaca@dimes.unical.it \\ 4 Istituto per le Applicazioni del Calcolo "Mauro Picone" (IAC), CNR, 80131 Naples, Italy; i.torcicollo@iac.cnr.it \\ * Correspondence: giuseppe.ali@unical.it
}

Citation: Alì, G.; Lamonaca, F.;

Scuro, C.; Torcicollo, I. On a

One-Dimensional Hydrodynamic

Model for Semiconductors with

Field-Dependent Mobility.

Mathematics 2021, 9, 2152.

https://doi.org/10.3390/

math9172152

Academic Editor: Arturo Hidalgo

Received: 4 August 2021

Accepted: 1 September 2021

Published: 3 September 2021

Publisher's Note: MDPI stays neutral with regard to jurisdictional claims in published maps and institutional affiliations.

Copyright: (C) 2021 by the authors. Licensee MDPI, Basel, Switzerland. This article is an open access article distributed under the terms and conditions of the Creative Commons Attribution (CC BY) license (https:// creativecommons.org/licenses/by/ $4.0 /)$.

\begin{abstract}
We consider a one-dimensional, isentropic, hydrodynamical model for a unipolar semiconductor, with the mobility depending on the electric field. The mobility is related to the momentum relaxation time, and field-dependent mobility models are commonly used to describe the occurrence of saturation velocity, that is, a limit value for the electron mean velocity as the electric field increases. For the steady state system, we prove the existence of smooth solutions in the subsonic case, with a suitable assumption on the mobility function. Furthermore, we prove uniqueness of subsonic solutions for sufficiently small currents.
\end{abstract}

Keywords: subsonic solutions; unipolar semiconductor; saturation velocity; steady-state hydrodynamical model

\section{Introduction}

The hydrodynamic model for semiconductors was first introduced by Bløtekjær in 1970 [1]. It describes the dynamics of charged fluid particles, such as electrons and holes in semiconductor devices [2,3], and positively and negatively charged ions in plasmas [4]. The ruling equations are the Euler-Poisson equations, which in their scaled one-dimensional form, for a unipolar semiconductor, are:

$$
\left\{\begin{array}{l}
n_{t}+j_{x}=0 \\
j_{t}+\left(\frac{j^{2}}{n}+p(n)\right)_{x}=\phi_{x} n-\frac{j}{\mu} \\
\phi_{x x}=n-N(x) .
\end{array}\right.
$$

where $n(x, t)$ is the electron number density, $j(x, t)$ is the electron flux density, $\phi(x, t)$ is the electric potential, $p(n)$ is the pressure and $\mu$ is mobility. The mobility is related to the momentum relaxation time, and in (1) they coincide because of the scaling. The device domain is the $x$-interval $(0, v)$, where $v$ is the device length measured in terms of the Debye length, and $N=N(x)$ is the number density of the background ions (doping profile), with $N(x)>0$.

The steady-state version of this one-dimensional model has been extensively studied, starting from the existence result, and uniqueness for small currents, in the subsonic case [5]. The existence of transonic solutions, with subsonic constant doping profile, was addressed in [6], with an approach based on the construction of the orbits of the system in the electron density-electric field phase plane. The same approach was later used in [7] for a more general case. A different approach was used in [8] for the construction on transonic solutions with transonic shocks, by using the vanishing viscosity limit method. The same 
method was used in [9], with prescribed current and periodic boundary conditions. We also refer to [10] for a non-isentropic case. More recently, the existence of supersonic solutions was proven in [11], and the existence of solutions for sonic boundary conditions was studied in a series of papers [12-14].

The hydrodynamic system for semiconductors has been studied also in higher dimension and in the time-dependent case. We can refer to [15] for potential flow in three dimensions with a fully subsonic doping profile, and to [15-21] for more general analysis of the subsonic case. For supersonic flows, the existence and uniqueness of supersonic solutions in two and three dimensions was studied in $[22,23]$. The transonic solutions have also been studied in relation to the formation of shock waves [24-28].

In all the above results, the mobility, or momentum relaxation time, is either assumed constant or a function of $n, j$. In this paper, we consider the effect of an electric-field dependent mobility, $\mu=\mu(E)$, with $E=\phi_{x}$. A preliminary discussion of this problem has been given in [29], with a phase-plane approach. Here, we prove the existence of subsonic solutions to the semiconductor hydrodynamic model with field-dependent mobility, for sufficiently small currents. Moreover, we prove the uniqueness of solutions for small currents. Both proofs are inspired by the classical results in [5], with some substantial changes due to the particular nature of the source term considered in our case. In particular, at variance with the standard case, it is not obvious how to relate the current with the electric potential at the boundaries, so some additional introductory work is needed.

The plan of the paper is the following. After Section 2, where the steady-state semiconductor hydrodynamical model is introduced and the problem is stated, Section 3 is devoted to proving the existence of subsonic solutions of the system. Section 4 shows a uniqueness result for regular solutions of the problem, while Section 5 concludes the paper with a short summary of the obtained findings.

\section{Statement of the Problem}

We consider the steady-state, one-dimensional semiconductor hydrodynamical model:

$$
\left\{\begin{array}{l}
j_{x}=0, \\
\left(\frac{j^{2}}{n}+p(n)\right)_{x}=\phi_{x} n-\frac{j}{\mu}, \quad \text { with } x \in(0, v), \\
\phi_{x x}=n-N(x),
\end{array}\right.
$$

with the following boundary conditions:

$$
n(0)=n_{0}, \quad n(v)=n_{1}, \quad \phi(0)=0, \quad \phi(v)=\phi_{1} .
$$

The pressure depends on the electron density by the constitutive relation $p=p(n)$, which satisfies the condition

$$
n^{2} p^{\prime}(n) \text { is strictly monotonically increasing from } \mathbb{R}_{+} \text {onto } \mathbb{R}_{+} \text {. }
$$

A common choice for the pressure relation is $p(n)=k n^{\gamma}$, with $\gamma \geq 1, k>0$.

The mobility is of the form

$$
\frac{1}{\mu}=\alpha q\left(\phi_{x}\right), \text { with } q(E) \geq q_{0}>0,\left|q^{\prime}(E)\right|<c_{q} \text { for all } E \in \mathbb{R},
$$

with $\alpha, q_{0}, c_{q}$ positive constants. The above condition holds for the most used high-field mobility models, like the Caughey-Thomas [30] or the Canali model [31], which can be written as:

$$
\mu(E)=\frac{\mu_{0}}{\left(1+\left(|E| / E_{\mathcal{C}}\right)^{\beta}\right)^{\frac{1}{\beta}}},
$$


with $\mu_{0}$ low-field mobility, $E_{c}$ critical field value, related to the saturation velocity, and $\beta \geq 1$ a real exponent. After scaling, for this specific model we get

$$
q(E)=\left(1+\left(c_{q}|E|\right)^{\beta}\right)^{\frac{1}{\beta}}, \quad q^{\prime}(E)=\frac{q(E)\left(c_{q}|E|\right)^{\beta}}{E\left(1+\left(c_{q}|E|\right)^{\beta}\right)^{\prime}},
$$

which satisfies (5).

The doping profile $N(x)$ satisfies the condition:

$$
N(\cdot) \in L^{\infty}(0, v), \quad N(x)>0 \text { for all } x \in(0, v) .
$$

The first equation in (2) gives $j=$ constant. We are going to see whether we can prescribe $j$ instead of $\phi_{1}$, as in the case where the mobility - that is, the momentum relaxation time-is independent of the electric field. For regular solutions, we can expand the derivative on the left-hand side of the second equation in (2), divide by $n$ and obtain

$$
F(n, j)_{x}=\phi_{x}-j \alpha \frac{q\left(\phi_{x}\right)}{n},
$$

with

$$
F(n, j)=\frac{j^{2}}{2 n^{2}}+h(n), \quad h^{\prime}(n)=\frac{1}{n} p^{\prime}(n) .
$$

Integrating (8) over $(0, v)$ and using the boundary conditions (3), we obtain

$$
\phi_{1}=F\left(n_{1}, j\right)-F\left(n_{0}, j\right)+j \alpha \int_{0}^{v} \frac{q\left(\phi_{x}(x)\right)}{n(x)} \mathrm{d} x .
$$

On the other hand, using the third equation in (2), we can represent $\phi$ and $\phi_{x}$ as integral functionals of $n-N$, depending on the boundary data $\phi_{1}$. The result is

$$
\begin{aligned}
\phi(x) & =\frac{1}{v} \phi_{1} x+\int_{0}^{x} \mathcal{I}_{n-N}(y) \mathrm{d} y, \\
\phi_{x}(x) & =\frac{1}{v} \phi_{1}+\mathcal{I}_{n-N}(x),
\end{aligned}
$$

where the functional $\rho(x) \mapsto \mathcal{I}_{\rho}(x)$ is defined by

$$
\mathcal{I}_{\rho}(x):=\int_{0}^{x} \rho\left(x_{1}\right) \mathrm{d} x_{1}-\frac{1}{v} \int_{0}^{v}\left(\int_{0}^{x_{2}} \rho\left(x_{1}\right) \mathrm{d} x_{1}\right) \mathrm{d} x_{2} .
$$

For a later use, it is simple to see that

$$
\left|\mathcal{I}_{\rho}(x)\right| \leq 2 v^{\frac{1}{2}}\|\rho\| \leq 2 v\|\rho\|_{\infty}
$$

with $\|\cdot\|$ norm in $L^{2}(0, v),\|\cdot\|_{\infty}$ norm in $L^{\infty}(0, v)$. Using (12), the relation (9) can be written in the form

$$
G\left(j, \phi_{1}\right):=F\left(n_{1}, j\right)-F\left(n_{0}, j\right)-\phi_{1}+j \alpha \int_{0}^{v} \frac{q\left(\frac{1}{v} \phi_{1}+\mathcal{I}_{n-N}(x)\right)}{n(x)} \mathrm{d} x=0 .
$$

We can use the implicit function theorem to show that (14) defines implicitly $\phi_{1}$ as a function of $j$. First of all, we observe that

$$
G\left(0, \phi_{\mathrm{bi}}\right)=0 \text {, with } \phi_{\mathrm{bi}}:=F\left(n_{1}, 0\right)-F\left(n_{0}, 0\right),
$$


which represents the so-called "built-in potential". Then, we can compute

$$
\frac{\partial G}{\partial \phi_{1}}\left(0, \phi_{\mathrm{bi}}\right)=\left(-1+\frac{j \alpha}{v} \int_{0}^{v} \frac{q^{\prime}\left(\frac{1}{v} \phi_{1}+\mathcal{I}_{n-N}(x)\right)}{n(x)} \mathrm{d} x\right)_{\substack{j=0 \\ \phi_{1}=\phi_{\mathrm{bi}}}}=-1 \neq 0 .
$$

It follows that (14) defines implicitly a function $g: I \rightarrow J$, with $I \subset \mathbb{R}$ neighborhood of 0 and $J \subset \mathbb{R}$ neighborhood of $\phi_{\mathrm{bi}}$, such that $G(j, g(j))=0$ for all $j \in I$. Moreover, we have

$$
\begin{aligned}
\frac{\partial G}{\partial j}\left(0, \phi_{\mathrm{bi}}\right) & =\left(\frac{j}{n_{1}^{2}}-\frac{j}{n_{0}^{2}}+\alpha \int_{0}^{v} \frac{q\left(\frac{1}{v} \phi_{1}+\mathcal{I}_{n-N}(x)\right)}{n(x)} \mathrm{d} x\right)_{\substack{j=0 \\
\phi_{1}=\phi_{\mathrm{bi}}}} \\
& =\alpha \int_{0}^{v} \frac{q\left(\frac{1}{\nu} \phi_{\mathrm{bi}}+\mathcal{I}_{n-N}(x)\right)}{n(x)} \mathrm{d} x,
\end{aligned}
$$

which implies

$$
g^{\prime}(0)=-\frac{\frac{\partial G}{\partial j}\left(0, \phi_{\mathrm{bi}}\right)}{\frac{\partial G}{\partial \phi_{1}}\left(0, \phi_{\mathrm{bi}}\right)}=\alpha \int_{0}^{v} \frac{q\left(\frac{1}{v} \phi_{\mathrm{bi}}+\mathcal{I}_{n-N}(x)\right)}{n(x)} \mathrm{d} x>0,
$$

that is, the function $\phi_{1}=g(j)$ is locally increasing around $j=0$.

If we know an a priori bound of the type $0<\underline{n} \leq n(x) \leq \bar{n}$ for $x \in(0, v)$, using assumption (5), for positive $j$ we find

$$
\frac{\partial G}{\partial \phi_{1}}\left(j, \phi_{1}\right)=-1+\frac{j \alpha}{v} \int_{0}^{v} \frac{q^{\prime}\left(\frac{1}{v} \phi_{1}+\mathcal{I}_{n-N}(x)\right)}{n(x)} \mathrm{d} x<-1+j \alpha \frac{\mathcal{c}_{q}}{\underline{n}},
$$

which implies that the implicit function $\phi_{1}=g(j)$ can be extended to all positive values of $j$ such that

$$
\frac{j}{\underline{n}} \leq \frac{1}{\alpha c_{q}} .
$$

The constant $\frac{1}{\alpha c_{q}}$ represents the saturation velocity, which is encoded in the fielddependent mobility model.

The above discussion implies that (14) can be used to replace the boundary conditions for $\phi$ with the assignment of the current $j$, when $j$ is small enough. Then, we can differentiate (8) and use the third equation in (2) (Poisson's equation) to obtain

$$
F(n, j)_{x x}+j \alpha\left(\frac{q\left(\phi_{x}\right)}{n}\right)_{x}-n=-N(x),
$$

with $\phi_{x}$ given by (11), and $\phi_{1}$ related to $j$ by (14). Equation (18) is supplemented with boundary conditions

$$
n(0)=n_{0}, \quad n(v)=n_{1} .
$$

In conclusion, the original problems (2) and (3) can be replaced by the integraldifferential Equation (18) with boundary conditions (19), where the current $j$ is assigned. The resulting solution will still depend on the boundary value $\phi_{1}$, which will be adjusted so that (14) is satisfied.

\section{Existence of Subsonic Solutions}

In this section we prove an existence result for subsonic solutions of system (2). Before stating the main theorem, we clarify the notion of subsonic solution. For regular solutions, the higher order term in (18) is

$$
F(n, j)_{x x}=\left(\frac{\partial F}{\partial n}(n, j) n_{x}\right),
$$


so the equation is uniformly elliptic if we can ensure that

$$
\frac{\partial F}{\partial n}(n, j)=-\frac{j^{2}}{n^{3}}+\frac{1}{n} p^{\prime}(n)>0,
$$

that is, $n^{2} p^{\prime}(n)>j^{2}$. Condition (4) implies that there exists a unique minimal value $n_{\min }$, depending on $j$, such that condition (20) holds for all $n>n_{\min }$. As a function of $j$, the minimal value $n_{\min }$ is strictly decreasing, and for $j=0$ we have $n_{\min }=0$. Thus, we can conclude that Equation (18) is uniformly elliptic for $n \geq n^{*}>n_{\min }$, for some constant $n^{*}$ to be determined. This bound on the electron density can be interpreted by saying that the solution is subsonic, since it amounts to the condition $|j / n|<\left(p^{\prime}(n)\right)^{\frac{1}{2}}$, with $j / n$ mean electron velocity and $\left(p^{\prime}(n)\right)^{\frac{1}{2}}$ speed of sound.

As a last remark, we note that we can limit ourselves to study the case $j>0$, because the case $j<0$ can be recovered from the same system (2) after a transformation $x \mapsto v-x$.

Theorem 1. Let us assume (4), (5) and (7), and let $p^{\prime}(n)$ and $q^{\prime}(E)$ be continuous functions of their arguments. Let us denote

$$
\begin{array}{cc}
\underline{N}=\inf _{x \in(0, v)} N(x), \quad \bar{N}=\sup _{x \in(0, v)} N(x), \\
\underline{n}=\min \left\{n_{0}, n_{1}, \underline{N}\right\}, \quad \bar{n}=\max \left\{n_{0}, n_{1}, \bar{N}\right\} .
\end{array}
$$

For $j>0$, under the assumption

$$
n_{\min }<\underline{n}, \quad j<\frac{\underline{n}}{\alpha c_{q}},
$$

and for any $\phi_{1}$, problems (18) and (19) have a solution which satisfies

$$
\underline{n} \leq n(x) \leq \bar{N} .
$$

Moreover, $\phi_{1}$ can be chosen so that (9) holds.

Proof. The proof is based on the construction of a fixed point map $T$ which, to a function $\hat{n}$ with

$$
\underline{n} \leq \hat{n}(x) \leq \bar{n} \text { for all } x \in(0, v),
$$

associates the solution $n$ of the problem

$$
\begin{aligned}
& \left(\frac{\partial F}{\partial n}(\hat{n}, j) n_{x}\right)_{x}-j \alpha \frac{q(\hat{E})}{\hat{n}^{2}} n_{x}-\left(1-j \alpha \frac{q^{\prime}(\hat{E})}{\hat{n}}\right) n=-\left(1-j \alpha \frac{q^{\prime}(\hat{E})}{\hat{n}}\right) N(x), \\
& n(0)=n_{0}, \quad n(v)=n_{1},
\end{aligned}
$$

with $\hat{E}(x)=\frac{1}{v} \phi_{1}+\mathcal{I}_{\hat{n}-N}(x)$. We notice that (13) ensures that $\hat{E}$ is bounded, and so is $q(\hat{E})$ and $q^{\prime}(\hat{E})$. Thanks to the first assumption in (21), Equation (24) is a linear, uniformly elliptic equation, which admits a unique solution $n=T(\hat{n})$ in $H^{1}(0, v)$. Moreover, thanks to the second assumption in (21), we have

$$
1-j \alpha \frac{q^{\prime}(\hat{E})}{\hat{n}}>1-j \alpha \frac{c_{q}}{\underline{n}}>0,
$$

so the maximum principle implies that $n$ satisfies the bounds (22). Recalling the definition (12), and (23), it is not difficult to use (24) and obtain $H^{1}$-bounds for $n$ independent of $\hat{n}$. By the compact imbedding of $H^{1}(0, v)$ into $C^{0}([0, v])$, and by a standard continuity argument we can use Schauder's theorem to conclude the existence of a fixed point of the map $T$. 
All the steps of this proof can be followed if, in the definition of the map $T$, we choose $\phi_{1}$ as the minimum value greater than $\phi_{\mathrm{bi}}$ such that

$$
F\left(n_{1}, j\right)-F\left(n_{0}, j\right)-\phi_{1}+j \alpha \int_{0}^{v} \frac{q\left(\frac{1}{v} \phi_{1}+\mathcal{I}_{\hat{n}-N}(x)\right)}{\hat{n}(x)} \mathrm{d} x=0 .
$$

This concludes the proof.

\section{Uniqueness of Solutions}

Theorem 2. Under the same assumptions of Theorem 1, there exists a constant $j_{0}>0$ such that for any $j<j_{0}$ there exists at most one regular solution $n$ of problems (18), (19), (9) and (11), which satisfies the bound $n(x) \geq \underline{n}>n_{\min }$ for all $x \in(0, v)$.

Proof. Let $n_{1}$ and $n_{2}$ be two solutions of problems (18), (19), (9) and (11), such that $n_{i}(x) \geq$ $\underline{n}>n_{\min }$ for all $x \in(0, v), i=1,2$. Thus, we have

$$
\begin{aligned}
& F\left(n_{i}(x), j\right)_{x x}+j \alpha\left(\frac{q\left(E_{i}(x)\right)}{n_{i}(x)}\right)_{x}-n_{i}(x)=-N(x), \\
& F\left(n_{1}, j\right)-F\left(n_{0}, j\right)-\phi_{1, i}+j \alpha \int_{0}^{v} \frac{q\left(E_{i}(x)\right)}{n_{i}(x)} \mathrm{d} x=0,
\end{aligned}
$$

with

$$
E_{i}(x)=\frac{1}{v} \phi_{1, i}+\mathcal{I}_{n_{i}-N}(x), \quad i=1,2 .
$$

Subtracting the Equation (27) and introducing the variable $u(x)=n_{2}(x)-n_{1}(x)$, we obtain

$$
(a(x) u)_{x x}+j \alpha\left(\frac{q\left(E_{2}(x)\right)}{n_{2}(x)}-\frac{q\left(E_{1}(x)\right)}{n_{1}(x)}\right)_{x}-u=0,
$$

with

$$
a(x)=\int_{0}^{1} \frac{\partial F}{\partial n}\left(n_{1}(x)+\theta\left(n_{2}(x)-n_{1}(x)\right), j\right) \mathrm{d} \theta
$$

Moreover, from (28) we find

$$
\phi_{1,2}-\phi_{1,1}=j \alpha \int_{0}^{v}\left(\frac{q\left(E_{2}(x)\right)}{n_{2}(x)}-\frac{q\left(E_{1}(x)\right)}{n_{1}(x)}\right) \mathrm{d} x .
$$

Multiplying (30) by $a(x) u$ and integrating over $(0, v)$, we find

$$
\int_{0}^{v}\left|(a(x) u)_{x}\right|^{2} \mathrm{~d} x+j \alpha \int_{0}^{v}(a(x) u)_{x}\left(\frac{q\left(E_{2}\right)}{n_{2}}-\frac{q\left(E_{1}\right)}{n_{1}}\right) \mathrm{d} x+\int_{0}^{v} a(x) u^{2} \mathrm{~d} x=0 .
$$

We can estimate

$$
\begin{aligned}
\left|\frac{q\left(E_{2}\right)}{n_{2}}-\frac{q\left(E_{1}\right)}{n_{1}}\right| & \leq \frac{1}{n_{2}}\left|q\left(E_{2}\right)-q\left(E_{1}\right)\right|+\frac{q\left(E_{1}\right)}{n_{2} n_{1}}|u| \\
& =\frac{1}{n_{2}}\left|\int_{0}^{1} q^{\prime}\left(E_{1}+\theta\left(E_{2}-E_{1}\right)\right) \mathrm{d} \theta\right|\left|E_{2}-E_{1}\right|+\frac{q\left(E_{1}\right)}{n_{2} n_{1}}|u| \\
& \leq \frac{c_{q}}{\underline{n}}\left|E_{2}-E_{1}\right|+\frac{\left\|q\left(E_{1}\right)\right\|_{\infty}}{\underline{n}^{2}}|u| .
\end{aligned}
$$

On the other hand, from (29) and (13), we find

$$
\left|E_{2}-E_{1}\right| \leq \frac{1}{v}\left|\phi_{1,2}-\phi_{1,1}\right|+\left|\mathcal{I}_{u}(x)\right| \leq \frac{1}{v}\left|\phi_{1,2}-\phi_{1,1}\right|+2 v^{\frac{1}{2}}\|u\|,
$$


and using (32) we obtain

$$
\begin{aligned}
& \left|\phi_{1,2}-\phi_{1,1}\right| \leq j \alpha \int_{0}^{v}\left|\frac{q\left(E_{2}(x)\right)}{n_{2}(x)}-\frac{q\left(E_{1}(x)\right)}{n_{1}(x)}\right| \mathrm{d} x \\
& \quad \leq j \alpha \frac{c_{q}}{\underline{n}} \int_{0}^{v}\left|E_{2}-E_{1}\right| \mathrm{d} x+j \alpha \frac{\left\|q\left(E_{1}\right)\right\|_{\infty}}{\frac{n^{2}}{\|}} \int_{0}^{v}|u| \mathrm{d} x \\
& \quad \leq j \alpha \frac{c_{q}}{\underline{n}}\left|\phi_{1,2}-\phi_{1,1}\right|+j \alpha \frac{c_{q}}{\underline{n}}\left(2+\frac{\left\|q\left(E_{1}\right)\right\|_{\infty}}{c_{q} \underline{n} v}\right) v^{\frac{3}{2}}\|u\| .
\end{aligned}
$$

Recalling the second assumption in (21), we get

$$
\left|\phi_{1,2}-\phi_{1,1}\right| \leq \frac{j \alpha \frac{c_{q}}{\underline{n}}}{1-j \alpha \frac{c_{q}}{\underline{n}}}\left(2+\frac{\left\|q\left(E_{1}\right)\right\|_{\infty}}{c_{q} \underline{n} v}\right) v^{\frac{3}{2}}\|u\|=: c_{\phi}(j) v^{\frac{3}{2}}\|u\|,
$$

which, together with (34) yields

$$
\left|E_{2}-E_{1}\right| \leq\left(2+c_{\phi}(j)\right) v^{\frac{1}{2}}\|u\| .
$$

The constant $c_{\phi}(j)$ tends to 0 as $j$ tends to zero. Finally, we can estimate

$$
\begin{aligned}
& \left|\int_{0}^{v}(a(x) u)_{x}\left(\frac{q\left(E_{2}\right)}{n_{2}}-\frac{q\left(E_{1}\right)}{n_{1}}\right) \mathrm{d} x\right| \leq\left\|(a(x) u)_{x}\right\|\left\|\frac{q\left(E_{2}\right)}{n_{2}}-\frac{q\left(E_{1}\right)}{n_{1}}\right\| \\
& \quad \leq\left\|(a(x) u)_{x}\right\|\left(\frac{c_{q}}{\underline{n}}\left\|\left(2+c_{\phi}(j)\right) v^{\frac{1}{2}}\right\| u\|\|+\frac{\left\|q\left(E_{1}\right)\right\|_{\infty}}{\underline{n}^{2}}\|u\|\right) \\
& \quad \leq \frac{c_{q} v}{\underline{n}} c_{\phi}^{*}(j)\left\|(a(x) u)_{x}\right\|\|u\|,
\end{aligned}
$$

with

$$
c_{\phi}^{*}(j):=2+c_{\phi}(j)+\frac{\left\|q\left(E_{1}\right)\right\|_{\infty}}{c_{q} \underline{\underline{n}} v} \equiv \frac{1}{1-j \alpha \frac{c_{q}}{\underline{n}}}\left(2+\frac{\left\|q\left(E_{1}\right)\right\|_{\infty}}{c_{q} \underline{\underline{n}} v}\right) .
$$

The constant $c_{\phi}^{*}(j)$ tends to a strictly positive value as $j$ tends to zero. Moreover, we can write

$$
\begin{aligned}
\left\|(a(x) u)_{x}\right\|\|u\| & \leq \frac{1}{\underline{a}}\left\|(a(x) u)_{x}\right\|\left\|a(x)^{\frac{1}{2}} u\right\| \\
& \leq \frac{1}{2 \underline{a}}\left(\left\|(a(x) u)_{x}\right\|^{2}+\left\|a(x)^{\frac{1}{2}} u\right\|^{2}\right),
\end{aligned}
$$

with $\underline{a}=\min _{x \in(0, v)} a(x)>0$. Thus, using (37) and (38) in (33), we find

$$
\left(1-j \alpha \frac{c_{q} v}{\underline{n}} \frac{c_{\phi}^{*}(j)}{2 \underline{a}}\right)\left(\int_{0}^{v}\left|(a(x) u)_{x}\right|^{2} \mathrm{~d} x+\int_{0}^{v} a(x) u^{2} \mathrm{~d} x\right) \leq 0 .
$$

It is possible to find a constant $j_{0}>0$ such that

$$
1-j \alpha \frac{c_{q} v}{\underline{n}} \frac{c_{\phi}^{*}(j)}{2 \underline{a}}>0 \text { for all } 0 \leq j \leq j_{0}
$$

For instance, if $j \leq \frac{n}{2 \alpha c_{q}}$, we have $c_{\phi}^{*}(j) \leq 2 c_{\phi}^{*}(0)$, so we can choose

$$
j_{0}=\frac{\underline{n}}{2 \alpha c_{q}} \min \left\{1, \frac{2 \underline{a}}{c_{\phi}^{*}(0) v}\right\} .
$$


It follows that, for $j \leq j_{0}$, we have

$$
\int_{0}^{v}\left|(a(x) u)_{x}\right|^{2} \mathrm{~d} x+\int_{0}^{v} a(x) u^{2} \mathrm{~d} x=0,
$$

which implies that $u(x) \equiv 0$ and thus the thesis.

\section{Conclusions}

The hydrodynamic model for semiconductors describing the dynamics of charged fluid particles, such as electron and holes in semiconductor devices and ions in plasmas, has attracted the attention of many scholars. In such studies the mobility, or momentum relaxation time, is either assumed constant or a function of the electron number density and the electron flux density. Here, we have considered the effect of an electric field dependent mobility. This kind of mobility model was introduced in the literature after empirical studies, to take into account the occurrence of a saturation velocity, that is, a maximum electron mean velocity which is reached as the electric fields grows and tends to infinity in modulus. The second condition in (21), which plays a key role in both the existence and the uniqueness proof, expresses precisely the upper bound of the electron velocity given by the saturation velocity. In our case, the saturation velocity $v_{S}$ can be recovered as the high-field limit of the velocity $\frac{j}{n}=\mu\left(\phi_{x}\right) \phi_{x}$, which follows by equating to zero the right-hand side of $(1)_{2}$. We obtain

$$
v_{s}=\lim _{E \rightarrow \infty} \mu(E) E=\lim _{E \rightarrow \infty} \frac{E}{\alpha q(E)}=\lim _{E \rightarrow \infty} \frac{1}{\alpha q^{\prime}(E)}=\frac{1}{\alpha c_{q}} .
$$

This conclusion is accurate if we use the explicit form (6), while in general the condition (5) only yields a lower bound $\frac{1}{\alpha c_{q}}$ for $v_{s}$, unless we add specific hypothesis on the behaviour of $q^{\prime}(E)$ at infinity.

In this paper, we have examined a one-dimensional, isentropic, hydrodynamical model for a unipolar semiconductor, with the relaxation time depending on the electric field. In this framework, for the steady-state version of the problem, we have proved the existence and uniqueness of smooth solutions in the subsonic case for sufficiently small currents. This promising result can be considered as the basis for a further exploration of the implication of a field-dependent mobility on the solutions of the hydrodynamic model for semiconductors, not only in the subsonic case but also in the transonic and supersonic case, addressing the stability of the steady-state solution and the asymptotic behaviour of general solutions for long times. These will be the topics for subsequent papers.

Author Contributions: Conceptualization, G.A. and C.S.; methodology, G.A. and I.T.; formal analysis, G.A., F.L., C.S. and I.T.; writing-original draft preparation, G.A. All authors have read and agreed to the published version of the manuscript.

Funding: This research received no external funding.

Institutional Review Board Statement: Not applicable.

Informed Consent Statement: Not applicable.

Data Availability Statement: Not applicable.

Acknowledgments: This paper has been performed under the auspices of the G.N.F.M. of INdAM.

Conflicts of Interest: The authors declare no conflict of interest.

\section{References}

1. Bløtekjær, K. Transport equations for electrons in two-valley semiconductors. IEEE Trans. Electron Devices 1970, $17,38-47$. [CrossRef]

2. Jüngel, A. Quasi-Hydrodynamic Semiconductor Equations; Birkhäuser: Basel, Switzerland, 2001.

3. Markowich, P.; Ringhofer, C.; Schmeiser, C. Semiconductor Equations; Springer: Wien, Austria, 1990. 
4. Sitenko, A.; Malnev, V. Plasma Physics Theory; Chapman \& Hall: London, UK, 1995.

5. Degond, P.; Markowich, P.A. On a One-Dimensional Steady-State Hydrodynamic Model for Semiconductors. Appl. Math. Lett. 1990, 3, 25-29. [CrossRef]

6. Ascher, U.M.; Markowich, P.A.; Pietra, P.; Schmeiser, C. A Phase Plane Analysis of Transonic Solutions for the Hydrodynamic Semiconductor Model. Math. Model. Methods Appl. Sci. 1991, 1, 347-376. [CrossRef]

7. Rosini, M.D. A phase analysis of transonic solutions for the hydrodynamic semiconductor model. Quart. Appl. Math. 2005, 63, 251-268. [CrossRef]

8. Gamba, I.M. Stationary transonic solutions of a one-dimensional hydrodynamic model for semiconductors. Commun. Part. Differ. Equ. 1992, 17, 553-577.

9. Fang, W.; Ito, K. Steady-State Solutions of a One-Dimensional Hydrodynamic Model for Semiconductors. J. Differ. Equ. 1997, 133, 224-244. [CrossRef]

10. Amster, P.; Varela, M.P.B.; Jüngel, A.; Mariani, M.C. Subsonic Solutions to a One-Dimensional Non-isentropic Hydrodynamic Model for Semiconductors. J. Math. Anal. Appl. 2001, 258, 52-62. [CrossRef]

11. Peng, Y.; Violet, I. Example of supersonic solutions to a steady state Euler-Poisson system. Appl. Math. Lett. 2006, 19, 1335-1340. [CrossRef]

12. Li, J.Y.; Mei, M.; Zhang, G.J.; Zhang, K.J. Steady hydrodynamic model of semiconductors with sonic boundary: (I) Subsonic doping profile. SIAM J. Math. Anal. 2017, 49, 4767-4811. [CrossRef]

13. Li, J.Y.; Mei, M.; Zhang, G.J.; Zhang, K.J. Steady hydrodynamic model of semiconductors with sonic boundary: (II) Supersonic doping profile. SIAM J. Math. Anal. 2018, 50, 718-734. [CrossRef]

14. Chen, L.; Mei, M.; Zhang, G.J.; Zhang, K.J. Steady hydrodynamic model of semiconductors with sonic boundary and transonic doping profile. J. Differ. Equ. 2020, 269, 8173-8211. [CrossRef]

15. Degond, P.; Markowich, P. A steady state potential flow model for semiconductors. Ann. Mater. Pura Appl. 1993, 4, 87-98. [CrossRef]

16. Bae, M.; Duan, B.; Xie, C. Subsonic solutions for steady Euler-Poisson system in two-dimensional nozzles. SIAM J. Math. Anal. 2014, 46, 3455-3480. [CrossRef]

17. Bae, M.; Duan, B.; Xie, C. Subsonic flow for the multidimensional Euler-Poisson system. Arch. Ration. Mech. Anal. 2016, 220, 155-191. [CrossRef]

18. Huang, F.; Mei, M.; Wang, Y.; Yu, H. Asymptotic convergence to stationary waves for unipolar hydrodynamic model of semiconductors. SIAM J. Math. Anal. 2011, 43, 411-429. [CrossRef]

19. Huang, F.; Mei, M.; Wang, Y.; Yu, H. Asymptotic convergence to planar stationary waves for multi-dimensional unipolar hydrodynamic model of semiconductors. J. Differ. Equ. 2011, 251, 1305-1331. [CrossRef]

20. Li, H.-L.; Markowich, P.; Mei, M. Asymptotic behavior of solutions of the hydrodynamic model of semiconductors. Proc. R. Soc. Edinb. Sect. A 2002, 132, 359-378. [CrossRef]

21. Nishibata, S.; Suzuki, M. Asymptotic stability of a stationary solution to a hydrodynamic model of semiconductors. Osaka J. Math. 2007, 44, 639-665. [CrossRef]

22. Bae, M.; Duan, B.; Xiao, J.; Xie, C. Structural Stability of Supersonic Solutions to the Euler-Poisson System. Arch. Ration. Mech. Anal. 2021, 239, 679-731. [CrossRef]

23. Bae, M.; Park, H. Three-dimensional flows of Euler-Poisson system for potential flow. Commun. Pure Appl. Anal. 2021, 20, 2421-2440. [CrossRef]

24. Bae, M.; Park, H. Radial transonic shock solutions of Euler-Poisson system in convergent nozzles. Discret. Contin. Dyn. Syst. S 2018, 11, 773-791. [CrossRef]

25. Duan, B.; Zhou, Y. Non-isentropic multi-transonic solutions of Euler-Poisson system. J. Differ. Equ. 2020, 268, 7029-7046. [CrossRef]

26. Gamba, I.M.; Morawetz, C.S. A viscous approximation for a 2-D steady semiconductor or transonic gas dynamic flow: Existence for potential flow. Commun. Pure Appl. 1996, 49, 999-1049. [CrossRef]

27. Luo, T.; Rauch, J.; Xie, C.; Xin, Z. Stability of transonic shock solutions for one-dimensional Euler-Poisson equations. Arch. Ration. Mech. Anal. 2011, 202, 787-827. [CrossRef]

28. Luo, T.; Xin, Z. Transonic shock solutions for a system of Euler-Poisson equations. Commun. Math. Sci. 2012, 10, 419-462. [CrossRef]

29. Alì, G.; Carini, M. A hydrodynamic model for semiconductors with field-dependent mobility. In Proceedings of the 14th Conference on Waves and Stability in Continuous Media (WASCOM 2007), Baia Samuele, Italy, 30 June-7 July 2007; Manganaro, N., Monaco, G., Rionero, S., Eds.; World Scientific: Singapore, 2008; pp. 13-18.

30. Caughey, D.M.; Thomas, R.E. Carrier Mobilities in Silicon Empirically Related to Doping and Field. Proc. IEEE 1967, 55, 2192-2193. [CrossRef]

31. Canali, C.; Majni, G.; Minder, R.; Ottaviani, G. Electron and Hole Drift Velocity Measurements in Silicon and Their Empirical Relation to Electric Field and Temperature. IEEE Trans. Electron Devices 1975, 22, 1045-1047. [CrossRef] 